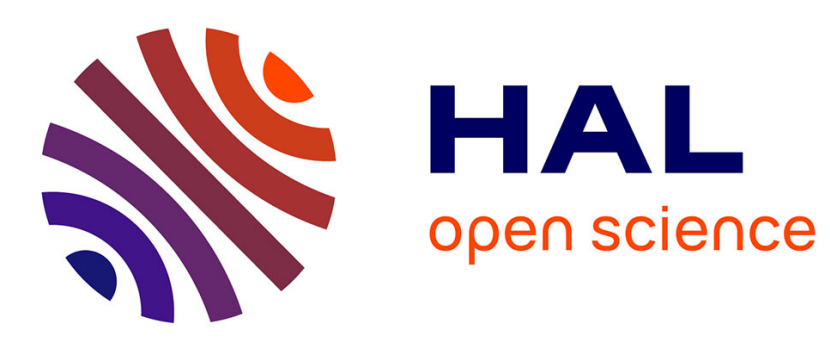

\title{
Maximum likelihood estimation for dynamic factor models with missing data
}

\author{
B. Jungbacker, S.J. Koopman, M. van Der Wel
}

\section{To cite this version:}

B. Jungbacker, S.J. Koopman, M. van Der Wel. Maximum likelihood estimation for dynamic factor models with missing data. Journal of Economic Dynamics and Control, 2011, 10.1016/j.jedc.2011.03.009 . hal-00828980

\section{HAL Id: hal-00828980 \\ https://hal.science/hal-00828980}

Submitted on 1 Jun 2013

HAL is a multi-disciplinary open access archive for the deposit and dissemination of scientific research documents, whether they are published or not. The documents may come from teaching and research institutions in France or abroad, or from public or private research centers.
L'archive ouverte pluridisciplinaire HAL, est destinée au dépôt et à la diffusion de documents scientifiques de niveau recherche, publiés ou non, émanant des établissements d'enseignement et de recherche français ou étrangers, des laboratoires publics ou privés. 


\section{Author's Accepted Manuscript}

Maximum likelihood estimation for dynamic factor models with missing data

B. Jungbacker, S.J. Koopman, M. van der Wel

PII: $\quad$ S0165-1889(11)00054-6

DOI: $\quad$ doi:10.1016/j.jedc.2011.03.009

Reference: DYNCON2555

To appear in: $\quad$ Journal of Economic Dynamics

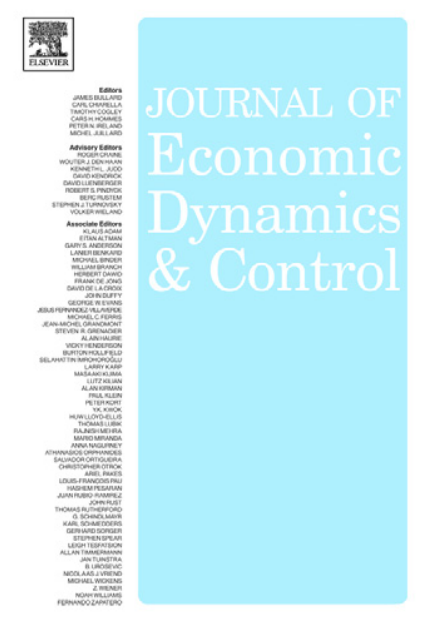

www.elsevier.com/locate/jedc \& Control

Received date: 14 January 2010

Accepted date: 18 March 2011

Cite this article as: B. Jungbacker, S.J. Koopman and M. van der Wel, Maximum likelihood estimation for dynamic factor models with missing data, Journal of Economic Dynamics \& Control, doi:10.1016/j.jedc.2011.03.009

This is a PDF file of an unedited manuscript that has been accepted for publication. As a service to our customers we are providing this early version of the manuscript. The manuscript will undergo copyediting, typesetting, and review of the resulting galley proof before it is published in its final citable form. Please note that during the production process errors may be discovered which could affect the content, and all legal disclaimers that apply to the journal pertain. 


\title{
Maximum likelihood estimation for dynamic factor models with missing data
}

\author{
B. Jungbacker ${ }^{(a)}$, S. J. Koopman ${ }^{(a, b) *}$ and M. van der Wel $l^{(b, c)}$ \\ (a) Department of Econometrics, VU University Amsterdam \\ (b) Tinbergen Institute \\ (c) Econometric Institute, Erasmus University Rotterdam and CREATES
}

March 24, 2011

\begin{abstract}
This paper concerns estimating parameters in a high-dimensional dynamic factor model by the method of maximum likelihood. To accommodate missing data in the analysis, we propose a new model representation for the dynamic factor model. It allows the Kalman filter and related smoothing methods to evaluate the likelihood function and to produce optimal factor estimates in a computationally efficient way when missing data is present. The implementation details of our methods for signal extraction and maximum likelihood estimation are discussed. The computational gains of the new devices are presented based on simulated data sets with varying numbers of missing entries.
\end{abstract}

JEL classification: C33; C43.

Some keywords: High-dimensional vector series; Kalman filtering and smoothing; Unbalanced panels of time series.

\section{Introduction}

This paper presents new procedures for parameter estimation by maximum likelihood and signal extraction of factors for high-dimensional factor models in the presence of missing data. We develop new methods for the basic dynamic factor model

$$
y_{t}=\Lambda f_{t}+u_{t}, \quad t=1, \ldots, n,
$$

\footnotetext{
${ }^{*}$ Corresponding author: S.J. Koopman, Department of Econometrics, VU University Amsterdam, De Boelelaan 1105, NL-1081 HV Amsterdam, The Netherlands. Email: s.j.koopman@feweb.vu.nl.
} 
where $y_{t}$ is the $N \times 1$ vector of time series observations, $f_{t}$ is an unobserved $q \times 1$ vector of common factors and $u_{t}$ is the $N \times 1$ vector of idiosyncratic terms. We focus on cases where $N$ is much larger than $q$. The factors are assumed to follow a Gaussian dynamic linear process and the idiosyncratic components in $u_{t}$ are modelled as autoregressive (AR) processes. The results are also applicable for more general models of this form.

In many economic applications, the dimension of $y_{t}$ is large and the model depends on a large number of parameters. The task of signal extraction and parameter estimation is therefore challenging in various respects. Particularly, state space formulations allow to obtain minimum mean square estimates of the factors together with the corresponding mean square errors by means of the Kalman filter and smoother recursions. The methods can be implemented in a computationally efficient way. However, the model representations are not valid in the presence of missing data. In empirical work, unbalanced panels for time series are more common than balanced panels without missing entries. For example, missings are present in the Stock and Watson (2002b) data set of 215 monthly time series and in the Otrok and Pourpourides (2008) longitudinal data set of hourly wages. Due to the existence of many data sets with missing entries we regard the problem of missing data as highly relevant.

We address the missing data problem by developing a low-dimensional linear state space model with time-varying state dimensions. It is equivalent to the dynamic factor model (1) and is designed to allow for missing entries in the dataset. We also discuss how the state space formulation can be used to obtain maximum likelihood estimates of the parameters by means of the Expectation-Maximization (EM) algorithm of Dempster, Laird, and Rubin (1977) and by direct optimization using a Quasi-Newton scheme.

Traditionally models of the form (1) have been estimated using principal components analysis (PCA), see Forni, Hallin, Lippi, and Reichlin (2000), Bai (2003) and Stock and Watson (2002a). Stock and Watson (2002b) is especially relevant as they show how missing data can be handled in a principal components analysis by means of an EM type algorithm. Sargent and Sims (1977) and Geweke (1977) are the earliest references discussing maximum likelihood methods for dynamic factor models. For a relatively low-dimensional model for wage rates, Engle and Watson (1981) consider maximum likelihood estimation, using Fisher scoring to maximize the likelihood. EM algorithms are developed for maximum likelihood estimation of parameters in state space models by Watson and Engle (1983) and Shumway and Stoffer (1982). Other estimation methods are based on the Bayesian approach as in Otrok and Whiteman (1998) and the subspace algorithm of Kapetanios and Marcellino (2009).

Recently there has been a renewed interest in the use of maximum likelihood estimation for high dimensional models. Doz, Giannone, and Reichlin (2010) show that estimates of the unobserved factors obtained from a likelihood-based analysis are consistent estimators of 
$f_{1}, \ldots, f_{n}$ as $n, N \rightarrow \infty$, even if the dynamic factor model is misspecified. Furthermore, they present evidence that in some cases a likelihood-based analysis produces more precise estimates of the factors than a principal component method. Reis and Watson (2010) consider the dynamic factor model (1) and obtain parameter estimates by the method of maximum likelihood using the approach of Watson and Engle (1983).

This maximum likelihood approach is not applicable when missing data is present. Banbura and Modugno (2010) propose a solution that overcomes the problem but is computationally demanding. Furthermore, their method requires numerical modifications to let the methods work in a satisfactory way. These modifications may distort the purpose of finding exact maximum likelihood estimates. In this paper we address the same issue but we develop a computationally efficient method for exact maximum likelihood parameter estimation. The methods also provide minimum mean square estimates and corresponding mean square errors of the factors and the idiosyncratic components.

The remainder of the paper is organized as follows. The dynamic factor model and its state space representations are discussed in detail in section 2. We develop in section 3 a new representation of the model that is valid when missing data is present. This state space representation allows the computationally efficient application of the Kalman filter and smoother recursions. Signal extraction and likelihood evaluation are explored in section 4. Parameter estimation by maximum likelihood methods are discussed in section 5 while computational comparisons based on simulated data is presented in section 6 . A short discussion of the presented results is given in section 7 .

\section{The dynamic factor model}

\subsection{Model specification}

The dynamic factor model given in (1) links the observation $y_{t}$ to a set of unobserved factors $f_{t}$ for $t=1, \ldots, n$. We assume that $f_{1}, \ldots, f_{n}$ are linear combinations of an unobserved $p \times 1$ dimensional vector autoregressive process $\alpha_{t}$ which is driven by $k \times 1$ dimensional disturbance vectors $\eta_{t}$ for $t=1, \ldots, n$. Specifically, there is a $q \times p$ selection matrix $S$ that defines the dynamic factor as

$$
f_{t}=S \alpha_{t}, \quad t=1, \ldots, n,
$$

and there is a transition equation for the state vector $\alpha_{t}$ as given by

$$
\alpha_{t+1}=T \alpha_{t}+R \eta_{t}, \quad \quad \eta_{t} \sim N\left(0, \Sigma_{\eta}\right), \quad t=1, \ldots, n,
$$


with the initial state vector $\alpha_{1}$ specified by $\alpha_{1} \sim N\left(0, \Sigma_{\alpha}\right)$ and where the $p \times p$ transition matrix $T$, the $p \times k$ disturbance selection matrix $R$, and the $k \times k$ variance matrix $\Sigma_{\eta}$ are assumed fixed (non-stochastic). The matrix $S$ is treated as a known selection matrix while matrices $T$ and $\Sigma_{\eta}$ may depend on a fixed and vector of unknown coefficients $\theta$. In case $\alpha_{t}$ is a time-invariant stationary process, the relation $\Sigma_{\alpha}=T \Sigma_{\alpha} T^{\prime}+\Sigma_{\eta}$ applies such that a solution for the (initial) state variance $\Sigma_{\alpha}$ exists when matrices $T$ and $\Sigma_{\eta}$ are given.

The dynamic specification for $f_{t}$ is general within the class of linear time series processes. For example, when the $p$ factors in $f_{t}$ are modeled as autoregressive moving average processes, the state vector consists of auxiliary variables that impose the serial dependence for the factors. The state vector dimension becomes typically much larger when more lags of $f_{t}$ are required to model the serial dependence. The matrix $S$ is a selection matrix of mostly zero and unity values; it provides the link between the variables in the possibly large dimensional state vector to the typically low dimensional $f_{t}$. The basic example is to have $f_{t}=\alpha_{t}$ so that $p=q$ and $S=I$. A more elaborate example is given in Appendix A to further illustrate the role of matrix $S$ in our framework. Other dynamic specifications for $f_{t}$ can also be considered and represented by (2) and (3). Further discussions of representing multivariate dynamic processes in state space are given by Shumway and Stoffer (2000, Chapter 6).

The dynamic factor model (1) can be expressed in terms of the state vector directly using the observation equation

$$
y_{t}=Z \alpha_{t}+u_{t}, \quad t=1, \ldots, n,
$$

where $Z=\Lambda S$. The factor loading matrix $\Lambda$ is treated as fixed and it depends on coefficient vector $\theta$. The idiosyncratic component $u_{t}$ is modelled as a vector autoregressive process with $r$ lags as given by

$$
u_{t+1}=\phi_{1} u_{t}+\cdots+\phi_{r} u_{t-r+1}+\varepsilon_{t}, \quad \varepsilon_{t} \sim N\left(0, \Sigma_{\varepsilon}\right)
$$

where $\phi_{1}, \ldots, \phi_{r}$ and $\Sigma_{\varepsilon}$ are $N \times N$ matrices. In general, $\phi_{1}, \ldots, \phi_{r}$ and the variance matrix of the initial vector $\left(u_{1}^{\prime}, \ldots, u_{r}^{\prime}\right)^{\prime}$ will be chosen such that $u_{t}$ is a stationary process. In case of $r=1$, the solution is implied by having the roots of $\left|\phi_{1}\right|=0$ outside the unit circle and by having the initial vector distributed as $u_{1} \sim N\left(0, \Sigma_{u}\right)$ with $\Sigma_{u}=\phi_{1} \Sigma_{u} \phi_{1}^{\prime}+\Sigma_{\varepsilon}$.

In the remainder of the paper, we consider the dynamic factor model as specified above. However, our results below apply to more general settings. We discuss these generalizations in some detail in section 3.3. 


\subsection{Two state space representations}

The dynamic factor model specification (4), (3) and (5) is close to the well-known state space model formulations of Harvey (1989) and Durbin and Koopman (2001). The Kalman filter and smoothing methods produce estimates of the state vector $\alpha_{t}$ with minimum mean square linear properties. In our formulation of a linear Gaussian dynamic factor model, we obtain minimum mean square estimates, see the discussions in Duncan and Horn (1972) and Anderson and Moore (1979). However, these optimal properties only apply when the observation equation (4) has disturbances $u_{t}$ that are not serially correlated. We can reformulate the dynamic factor model in two ways to ensure that the optimal properties of the Kalman filter and smoothing methods are preserved. The two formulations are given as $\mathrm{A}$ and $\mathrm{B}$ below for the special case of $r=1$. The higher-order case of $r>1$ follows straightforwardly but is notationally more cumbersome.

A. A basic approach is to express the dynamic factor model in terms of $\left(1-\phi_{1} L\right) y_{t}$ where $L$ is the lag-operator. When the polynomial function $1-\phi_{1} L$ is applied to both sides of (4), we obtain

$$
\begin{aligned}
y_{t} & =\phi_{1} y_{t-1}+Z \alpha_{t}-\phi_{1} Z \alpha_{t-1}+\varepsilon_{t} \\
& =c_{t}+\left(Z,-\phi_{1} Z\right)\left(\begin{array}{c}
\alpha_{t} \\
\alpha_{t-1}
\end{array}\right)+\varepsilon_{t},
\end{aligned}
$$

with $y_{1}=Z \alpha_{1}+u_{1}$ and where $c_{t}=\phi_{1} y_{t-1}$ for $t=2, \ldots, n$. The transition equation for the augmented state vector is given by

$$
\left(\begin{array}{c}
\alpha_{t+1} \\
\alpha_{t}
\end{array}\right)=\left[\begin{array}{cc}
T & 0 \\
I & 0
\end{array}\right]\left(\begin{array}{c}
\alpha_{t} \\
\alpha_{t-1}
\end{array}\right)+\left(\begin{array}{c}
\eta_{t} \\
0
\end{array}\right)
$$

for $t=1, \ldots, n$. The introduction of $c_{t}$ in the observation equation does not cause further complications; it can be incorporated in the Kalman filter since $c_{t}$ is known at time $t$.

B. An alternative formulation is obtained by augmenting the state vector with $u_{t}$ and is given by

$$
y_{t}=(Z, I)\left(\begin{array}{c}
\alpha_{t} \\
u_{t}
\end{array}\right), \quad\left(\begin{array}{c}
\alpha_{t+1} \\
u_{t+1}
\end{array}\right)=\left[\begin{array}{cc}
T & 0 \\
0 & \phi_{1}
\end{array}\right]\left(\begin{array}{c}
\alpha_{t} \\
u_{t}
\end{array}\right)+\left(\begin{array}{l}
\eta_{t} \\
\varepsilon_{t}
\end{array}\right),
$$

for $t=1, \ldots, n$. The initial condition for the state vector process is straightforwardly determined. The observation disturbance vector has disappeared from this formulation. This loss does not cause complications in the application of the Kalman filter. 
Both formulations will lead to the same results when initialisation issues are properly accounted for. Watson and Engle (1983) and, more recently, Reis and Watson (2010) have adopted formulation A while Banbura and Modugno (2010) have adopted formulation B.

\subsection{Missing data}

In this paper we are concerned with the application of the Kalman filter and smoothing methods to the dynamic factor model in the presence of missing observations. We assume that observations are missing at random and that the model is also applicable to missing data. The model formulation $\mathrm{B}$ is valid when $y_{t}$ contains missing entries while formulation $\mathrm{A}$ is not valid since $c_{t}$ cannot be determined when $y_{t-1}$ is partly missing. An exact treatment of filtering and smoothing is therefore not possible when we adopt formulation $\mathrm{A}$. The replacement of $c_{t}$ by $\hat{c}_{t}=\phi_{1} E\left(y_{t} \mid y_{1}, \ldots, y_{t-1}\right)$ in the Kalman filter applied to formulation A may lead to a practical solution but it clearly does not lead to an exact solution. This assessment has led Banbura and Modugno (2010) to adopt formulation B for their dynamic factor analysis. The solution is however computationally inefficient since the dimension of the enlarged state vector $\left(\alpha_{t}^{\prime}, u_{t}^{\prime}\right)^{\prime}$ will become very large when $N$ increases. In the empirical study of Banbura and Modugno (2010), the observation dimension is close to $N=100$ such that their state vector dimension is larger than 100. Although this solution is applicable, a huge dimensional state vector slows down the Kalman filter enormously and may even lead to numerical inaccuracies. It further prohibits its application in a routine manner. Therefore we prefer formulation A where the increase of the state dimension is moderate. The main contribution of this paper is a re-formulation of A that enables Kalman filter and smoothing methods to produce optimal estimates in the presence of missing data. Furthermore we show that the recent developments reported in Jungbacker and Koopman (2008) can be exploited as well in the new state space formulation given below.

\section{State space formulation in presence of missing data}

In this section we will show how the model of Section 2 can be written as a Gaussian state space model. For ease of notation we pursue the special case of $r=1$ and a diagonal coefficient matrix $\phi_{1}$. Section 3.3 discusses the consequences of more general model specifications.

\subsection{Notation}

Consider some $N \times 1$ vector $v_{t}$. The vector $v_{t}\left(o_{s}\right)$ contains all elements of $v_{t}$ that correspond to observed entries in the $N \times 1$ data vector $y_{s}$ for $t, s=1, \ldots, n$. In a similar way, $v_{t}\left(m_{s}\right)$ contains all elements of $v_{t}$ that correspond to missing entries in $y_{s}$. In case all entries in $y_{s}$ are observed, $v_{t}\left(m_{s}\right)$ is an empty vector. The vector $v_{t}\left(o_{s}, m_{s^{\prime}}\right)$ contains all elements of $v_{t}$ that 
correspond only to observed entries in $y_{s}$ and missing entries in $y_{s^{\prime}}$ for $t, s, s^{\prime}=1, \ldots, n$. Using this notation we can split the vector $v_{t}$ into four mutually exclusive subvectors $v_{t}\left(o_{s}, o_{s^{\prime}}\right)$, $v_{t}\left(o_{s}, m_{s^{\prime}}\right), v_{t}\left(m_{s}, o_{s^{\prime}}\right)$ and $v_{t}\left(m_{s}, m_{s^{\prime}}\right)$. In case we have no missing data, vectors $v_{t}\left(m_{s}\right)$ and $v_{t}\left(m_{s}, m_{s^{\prime}}\right)$ are empty while $v_{t}=v_{t}\left(o_{s}\right)=v_{t}\left(o_{s}, o_{s^{\prime}}\right)$. We further note that

$$
\left\{v_{t}\right\}=\left\{v_{t}\left(o_{s}\right), v_{t}\left(m_{s}\right)\right\}=\left\{v_{t}\left(o_{s}, o_{s^{\prime}}\right), v_{t}\left(o_{s}, m_{s^{\prime}}\right), v_{t}\left(m_{s}, o_{s^{\prime}}\right), v_{t}\left(m_{s}, m_{s^{\prime}}\right)\right\}
$$

To illustrate the notation, consider $N=5$ and

$$
y_{t}=(1, m, 2, m, 3)^{\prime}, \quad y_{t-1}=(m, m, m, 4,5)^{\prime}, \quad v_{t}=(6,7,8,9,10)^{\prime}
$$

where $m$ denotes a missing entry. It follows that

$$
\begin{aligned}
& v_{t}\left(o_{t}\right)=(6,8,10)^{\prime}, \quad v_{t}\left(m_{t}\right)=(7,9)^{\prime}, \\
& v_{t}\left(o_{t}, o_{t-1}\right)=10, \quad v_{t}\left(o_{t}, m_{t-1}\right)=(6,8)^{\prime}, \quad v_{t}\left(m_{t}, o_{t-1}\right)=9, \quad v_{t}\left(m_{t}, m_{t-1}\right)=7,
\end{aligned}
$$

The notation applies to matrices in a similar way. Consider the $N \times h$ matrix $V$. Matrix $V\left(o_{t} ; \cdot\right)$ contains selected rows of $V$ that correspond to the observed entries in $y_{t}$ while all columns are retained. In case of an $h \times N$ matrix $V$, the selection $V\left(\cdot ; o_{t}\right)$ applies to columns. In case of an $N \times N$ matrix $V$, the selection $V\left(o_{t} ; m_{t}\right)$ applies to both rows and columns.

\subsection{The missing data state space formulation}

Consider the model equations (4), (5) and (3). We develop a modified state space formulation for the observation vector with only observed entries, that is

$$
y_{t}^{o}=\left(\begin{array}{c}
y_{t}\left(o_{t}, o_{t-1}\right) \\
y_{t}\left(o_{t}, m_{t-1}\right)
\end{array}\right)
$$

for $t=1, \ldots, n$. We accomplish the formulation on the basis of the augmented state vector

$$
\dot{\alpha}_{t}=\left[\alpha_{t}^{\prime}, \alpha_{t-1}^{\prime}, u_{t}\left(o_{t}, m_{t-1}\right)^{\prime}, u_{t}\left(m_{t}, m_{t-1}\right)^{\prime}, u_{t}\left(m_{t}, o_{t-1}\right)^{\prime}\right]^{\prime}
$$

The state vector is augmented with its lagged counterpart $\alpha_{t-1}$ and with a selection of the idiosyncratic component $u_{t}$. The new formulation below can therefore be interpreted as a mix of formulations $\mathrm{A}$ and $\mathrm{B}$ in section 2.2. We require the subset $u_{t}\left(o_{t}, m_{t-1}\right)$ of $u_{t}$ to incorporate them in the observed entries of $y_{t}$ as implied by (4). The updating of $u_{t}$ to $u_{t+1}$ as implied by (5), with $r=1$, requires also the remaining $u_{t}$ entries, that is $u_{t}\left(m_{t}\right)=\left[u_{t}\left(m_{t} ; m_{t-1}\right)^{\prime}, u_{t}\left(m_{t} ; o_{t-1}\right)^{\prime}\right]^{\prime}$. The two entries in $u_{t}\left(m_{t}\right)$ are explicitly given in the augmented state vector $\dot{\alpha}_{t}$ because their treatments in the updating of $\dot{\alpha}_{t}$ to $\dot{\alpha}_{t+1}$ are different. The details are given next. 
The observation equation that links the observation vector $y_{t}^{o}$ and the state vector $\dot{\alpha}_{t}$ is obtained straightforwardly as

$$
y_{t}^{o}=c_{t}^{o}+\left[\begin{array}{ccccc}
Z\left(o_{t}, o_{t-1} ; \cdot\right) & -\phi_{t}^{o} Z\left(o_{t}, o_{t-1} ; \cdot\right) & 0 & 0 & 0 \\
Z\left(o_{t}, m_{t-1} ; \cdot\right) & 0 & I & 0 & 0
\end{array}\right] \dot{\alpha}_{t}+\left(\begin{array}{c}
\varepsilon_{t}\left(o_{t}, o_{t-1}\right) \\
0
\end{array}\right) \text {, }
$$

where $c_{t}^{o}=\left[\left\{\phi_{t}^{o} y_{t-1}\left(o_{t}, o_{t-1}\right)\right\}^{\prime}, 0\right]^{\prime}$ and $\phi_{t}^{o}=\phi_{1}\left(o_{t}, o_{t-1} ; o_{t}, o_{t-1}\right)$. Matrix $\phi_{t}^{o}$ is diagonal consisting of (a subset of), possibly reshuffled, diagonal elements of $\phi_{1}$. The specification for $y_{t}\left(o_{t}, o_{t-1}\right)$ relies on formulation A while for $y_{t}\left(o_{t}, m_{t-1}\right)$ it relies on formulation $\mathrm{B}$. The major difference of our formulation with $\mathrm{B}$ is that we only include those entries of $u_{t}$ in the state vector that correspond to missing entries in $y_{t}$ and/or $y_{t-1}$. For entries of $y_{t}$ where both $y_{t}$ and $y_{t-1}$ are observed, we compute the entries in $c_{t}^{o}$ and rely on formulation A.

The transition equation for the state process $\dot{\alpha}_{t}$ is obtained as follows. The updates for $\alpha_{t}$ and $\alpha_{t-1}$ are given as in (7) for formulation A. Next we develop equations for $u_{t+1}\left(o_{t+1}, m_{t}\right)$ and $u_{t+1}\left(m_{t+1}, m_{t}\right)$ which are effectively the selection $u_{t+1}\left(m_{t}\right)$ (re-ordered). The transition from $u_{t}\left(m_{t}\right)$ to $u_{t+1}\left(m_{t}\right)$ is the autoregressive update (5) with $r=1$ in our case. For our selection of $u_{t}$, we have

$$
u_{t+1}\left(m_{t}\right)=\phi_{1}\left(m_{t} ; m_{t}\right) u_{t}\left(m_{t}\right)+\varepsilon_{t}\left(m_{t}\right), \quad u_{t}\left(m_{t}\right)=\left(\begin{array}{c}
u_{t}\left(m_{t}, m_{t-1}\right) \\
u_{t}\left(m_{t}, o_{t-1}\right)
\end{array}\right),
$$

for $t=1, \ldots, n$. To place $u_{t+1}\left(m_{t}\right)$ into $\dot{\alpha}_{t+1}$, we need to re-order it into

$$
\left(\begin{array}{c}
u_{t+1}\left(o_{t+1}, m_{t}\right) \\
u_{t+1}\left(m_{t+1}, m_{t}\right)
\end{array}\right)=J_{t} u_{t+1}\left(m_{t}\right)
$$

where $J_{t}$ is implicitly defined as a selection matrix of ones and zeroes. The bottom part of $\dot{\alpha}_{t+1}$ is $u_{t+1}\left(m_{t+1}, o_{t}\right)$ and corresponds to observed entries in $y_{t}$. Therefore, we have

$$
\begin{aligned}
u_{t+1}\left(m_{t+1}, o_{t}\right) & =\phi_{t}^{*} u_{t}\left(m_{t+1}, o_{t}\right)+\varepsilon_{t}\left(m_{t+1}, o_{t}\right) \\
& =\phi_{t}^{*}\left[y_{t}\left(m_{t+1}, o_{t}\right)-Z_{t}^{*} \alpha_{t}\right]+\varepsilon_{t}\left(m_{t+1}, o_{t}\right),
\end{aligned}
$$

where $\phi_{t}^{*}=\phi_{1}\left(m_{t+1}, o_{t} ; m_{t+1}, o_{t}\right)$ and $Z_{t}^{*}=Z\left(m_{t+1}, o_{t} ; \cdot\right)$. The transition equation for $\dot{\alpha}_{t}$ is therefore

$$
\dot{\alpha}_{t+1}=d_{t}+\left[\begin{array}{cccc}
T & 0 & 0 & 0 \\
I & 0 & 0 & 0 \\
0 & 0 & 0 & J_{t} \phi_{1}\left(m_{t} ; m_{t}\right) \\
-\phi_{t}^{*} Z_{t}^{*} & 0 & 0 & 0
\end{array}\right]\left(\begin{array}{c}
\alpha_{t} \\
\alpha_{t-1} \\
u_{t}\left(o_{t}, m_{t-1}\right) \\
u_{t}\left(m_{t}\right)
\end{array}\right)+\left(\begin{array}{c}
\eta_{t} \\
0 \\
J_{t} \varepsilon_{t}\left(m_{t}\right) \\
\varepsilon_{t}\left(m_{t+1}, o_{t}\right)
\end{array}\right)
$$

where $d_{t}=\left[0,0,0,\left\{\phi_{t}^{*} y_{t}\left(m_{t+1}, o_{t}\right)\right\}^{\prime}\right]^{\prime}$, for $t=1, \ldots, n$. 


\subsection{Discussion of the new formulation}

The equations (9) and (10) define the state space model for the observed values while the missing observations are accounted for by including the relevant $u_{t}$ 's in the state vector. In case we have no missing data, the vectors $u_{t}\left(o_{t}, m_{t-1}\right)$ and $u_{t}\left(m_{t}\right)$ are empty and we return to formulation A. Entries of $u_{t}$ only appear in the state vector when they correspond to missing entries in $y_{t}$ or in $y_{t-1}$. In this way we keep the dimension of the state to a minimum while we are able to produce optimal estimates using Kalman filter and smoothing for all time periods. Further, the Markov property of the state space model implies that only the missing entries in $y_{t}$ and $y_{t-1}$ are relevant for our methods.

In most cases the dimension of $\dot{\alpha}_{t}$ will be smaller than the dimension of $\left(\alpha_{t}^{\prime}, u_{t}^{\prime}\right)^{\prime}$, the state vector in model formulation B. In case $\dot{\alpha}_{t}$ has a larger dimension than $\left(\alpha_{t}^{\prime}, u_{t}^{\prime}\right)^{\prime}$, due to a large number of missings in $y_{t-1}$ or $y_{t}$, it is possible to reduce the dimension of $\dot{\alpha}_{t}$ by dropping $\alpha_{t-1}$ (partially and temporarily) from the state vector $\dot{\alpha}_{t}$. This result follows because $\alpha_{t-1}$ is not required in the augmented state vector $\dot{\alpha}_{t}$ when all entries in $u_{t}$ are included in $\dot{\alpha}_{t}$. Computational gains are relatively small and we therefore do not pursue this further.

The new formulation does imply time-varying system matrices in the observation and transition equations. In fact, the dimension of the state vector also varies over time. Fortunately, the Kalman filter can treat varying dimensions for the state vector. The implementation of such a Kalman filter requires attention but it comes with the benefit of a dynamic factor analysis that is computationally feasible when missing data is present. We give some more details in the next section.

The results presented in this paper apply to more general settings. For example, the dynamic specification of $f_{t}$ may also depend on non-stationary time series processes such as a random walk. The Kalman filter and smoothing need to deal with the initialisation problem but existing solutions can be applied straightforwardly. Lagged factors and explanatory variables can be included in the observation equation of the dynamic factor model, see the discussion in Jungbacker and Koopman (2008). Given our solution, the case of $r>1$ is trivial but requires more notation in the expositions of sections 3 and 4. A particular concern is the case of a non-diagonal autoregressive coefficient matrix $\phi_{1}$ since it mixes the lag-dependence of idiosyncratic components associated with missing entries to those associated with observed entries. We therefore need to modify the system matrices in (10) accordingly. This exercise is straightforward but the notation is somewhat cumbersome.

\section{Signal extraction and likelihood evaluation}

In this section we discuss computationally efficient approaches to signal extraction and likelihood evaluation. These methods are also relevant for parameter estimation that is discussed in section 5 . 


\subsection{Estimation of states and idiosyncratic components}

Given the state space formulation of the dynamic factor model, we can adopt the Kalman filter and associating smoothing methods (KFS) to obtain

$$
\dot{a}_{t \mid s}=\mathbb{E}\left(\dot{\alpha}_{t} \mid Y_{s}\right), \quad \dot{P}_{t \mid s}=\operatorname{Var}\left(\dot{\alpha}_{t} \mid Y_{s}\right)
$$

for $t, s=1, \ldots, n$ where $Y_{s}=\left(y_{1}^{o}, \ldots, y_{s}^{o}\right)$, see Durbin and Koopman (2001) for an exposition of these methods. Prediction refers to $s=t-1$, concurrent filtering to $s=t$ and smoothing to $s=n$. The Kalman filter can also be used to evaluate the log-likelihood function using the prediction error decomposition result of Schweppe (1965), see section 4.4.

In terms of the dynamic factor model (4), (3) and (5), KFS produces estimates (as well as the mean square errors) of $\alpha_{t}$ and of those entries of $u_{t}$ that are associated with missing entries in $y_{t}$ and $y_{t-1}$, that is $u_{t}^{m}=\left[u_{t}\left(o_{t}, m_{t-1}\right)^{\prime}, u_{t}\left(m_{t}\right)^{\prime}\right]^{\prime}$. We can also obtain estimates and corresponding mean square errors of $u_{t}^{o}=u_{t}\left(o_{t}, o_{t-1}\right)$ using the identity $u_{t}=y_{t}-Z \alpha_{t}$ in (4). Let $a_{t \mid s}=\mathbb{E}\left(\alpha_{t} \mid Y_{s}\right)$ and $P_{t \mid s}=\operatorname{Var}\left(\alpha_{t} \mid Y_{s}\right)$ for $t, s=1, \ldots, n$. Obviously, $a_{t \mid s}$ and $P_{t \mid s}$ are the upper (block) parts of $\dot{a}_{t \mid s}$ and $\dot{P}_{t \mid s}$, respectively. It follows that

$$
\begin{aligned}
\mathbb{E}\left(u_{t}^{o} \mid Y_{s}\right) & =y_{t}^{o}-Z_{t}^{o} a_{t \mid s}, & \operatorname{Var}\left(u_{t}^{o} \mid Y_{s}\right) & =Z_{t}^{o} P_{t \mid s} Z_{t}^{o \prime}, \\
\operatorname{Cov}\left(u_{t}^{o}, \alpha_{t} \mid Y_{s}\right) & =-Z_{t}^{o} P_{t \mid s}, & \operatorname{Cov}\left(u_{t}^{o}, u_{t}^{m} \mid Y_{s}\right) & =-Z_{t}^{o} \operatorname{Cov}\left(\alpha_{t}, u_{t}^{m} \mid Y_{s}\right),
\end{aligned}
$$

where $Z_{t}^{o}=Z\left(o_{t}, o_{t-1} ; \cdot\right)$ and $\operatorname{Cov}\left(u_{t}^{m}, \alpha_{t} \mid Y_{s}\right)$ is part of $\dot{P}_{t \mid s}$ for $t, s=1, \ldots, n$.

\subsection{KFS with a collapsed observation vector}

The computational effort for the KFS depends on the dimensions of both the state and observation vectors. Consider the dynamic factor model (1) with $q \times 1$ vector $f_{t}=S \alpha_{t}$ and state space representation (4) and (3) but with $u_{t}$ replaced by $\varepsilon_{t} \sim N\left(0, \Sigma_{\varepsilon}\right)$, that is

$$
y_{t}=Z \alpha_{t}+\varepsilon_{t}, \quad \alpha_{t+1}=T \alpha_{t}+\eta_{t}
$$

for $t=1, \ldots, n$ with $N \times 1$ observation vector $y_{t}$ and $p \times 1$ state vector $\alpha_{t}$. In most practical applications of the dynamic factor model, the dimension of $y_{t}$ is significantly larger than the dimension of $\alpha_{t}$. Jungbacker and Koopman (2008) demonstrate that in such circumstances, when $N>q$, computational efficiency of KFS can significantly be improved by a simple computational device. We have $Z=\Lambda S$ and we define the $N \times N$ and $q \times N$ matrices

$$
A=\left[\begin{array}{l}
A^{L} \\
A^{H}
\end{array}\right], \quad A^{L}=C^{-1} \Lambda^{\prime} \Sigma_{\varepsilon}^{-1},
$$


respectively, where $C$ can be any $q \times q$ invertible matrix and $A^{H}$ is chosen such that matrix $A$ is full rank and $A^{L} \Sigma_{\varepsilon} A^{H \prime}=0$. It follows that $A^{H} \Lambda=0$. We assume that $\Lambda$ has full column rank. In most cases of practical interest this assumption will be valid. If matrix $\Lambda$ does not have full rank, it can be replaced with any full rank matrix that spans the column space of $\Lambda$, see the discussion in Jungbacker and Koopman (2008). Matrix $A^{H}$ exists by construction but it does not need to be evaluated for our purposes. By choosing $C$ such that $C C^{\prime}=\Lambda^{\prime} \Sigma_{\varepsilon}^{-1} \Lambda$, we have

$$
A y_{t}=\left(\begin{array}{c}
A^{L} y_{t} \\
A^{H} y_{t}
\end{array}\right)=\left(\begin{array}{c}
C^{\prime} S \\
0
\end{array}\right) \alpha_{t}+\left(\begin{array}{c}
A^{L} \varepsilon_{t} \\
A^{H} \varepsilon_{t}
\end{array}\right), \quad\left(\begin{array}{c}
A^{L} \varepsilon_{t} \\
A^{H} \varepsilon_{t}
\end{array}\right) \sim N\left(0,\left[\begin{array}{cc}
I & 0 \\
0 & A^{H} \Sigma_{\varepsilon} A^{H \prime}
\end{array}\right]\right)
$$

for $t=1, \ldots, n$. The equation for $\alpha_{t+1}$ is unaffected by the transformation. It follows

that the part $A^{H} y_{t}$ does not depend on $\alpha_{t}$, it is not correlated with $A^{L} y_{t}$ and therefore does not need to be considered for the estimation of $\alpha_{t}$. Therefore, the KFS only need to be applied to the collapsed observation (low-dimensional) vector $A^{L} y_{t}$ for signal extraction. Since $\operatorname{Var}\left(A^{L} \varepsilon_{t}\right)=I$, we can adopt the KFS devices discussed in Koopman and Durbin (2003) to further accelerate the computations.

The collapse can lead to high computational savings. To illustrate the reductions that we can achieve in practice, consider model (1) with $N=100$ and $q=5$. In this case, the observation vector relevant for the application of the KFS collapses from dimension $N=100$ to dimension $q=5$. Jungbacker and Koopman (2008) also demonstrate that likelihood evaluation can rely on the Kalman filter applied to $A^{L} y_{t}$, see section 4.4.

The presence of missing entries in the data set requires a further modification of the collapsed KFS. It is immediately obvious that the multiplication $A^{L} y_{t}$ returns a vector with all missing entries when at least one entry in $y_{t}$ is missing. In other words, the vector $A^{L} y_{t}$ does not exist when missing entries are present in $y_{t}$. The presented modification in the next section applies more generally; it is applicable to all multivariate time series models that can be represented in a linear state space form.

\subsection{A collapsed KFS in presence of missing data}

The computational device of Jungbacker and Koopman (2008) can be modified in the context of the state space formulation developed in section 3.2, in case missing data is present. Consider the observation equation (9). Since this formulation relies on time-varying system matrices, we require the collapsed transformations to vary over time as well.

We carry out a partial collapse of $y_{t}^{o}$ and only consider the transformation of $y_{t}\left(o_{t}, o_{t-1}\right)$ with dimension $N_{t}^{o o}$. For this purpose, we define

$$
A_{t}^{L}=C_{t}^{-1} Z_{t}^{+\prime} V_{t}^{-1}, \quad Z_{t}^{+}=\left[\Lambda\left(o_{t}, o_{t-1} ; \cdot\right),-\phi_{t}^{o} \Lambda\left(o_{t}, o_{t-1} ; \cdot\right)\right], \quad V_{t}=\Sigma_{\varepsilon}\left(o_{t}, o_{t-1} ; o_{t}, o_{t-1}\right)
$$


where $\phi_{t}^{o}=\phi_{1}\left(o_{t}, o_{t-1} ; o_{t}, o_{t-1}\right)$ and $C_{t}$ is chosen such that

$$
C_{t} C_{t}^{\prime}=Z_{t}^{+\prime} V_{t}^{-1} Z_{t}^{+}
$$

for $t=1, \ldots, n$. Again, we should make sure that $Z_{t}^{+}$has full column rank. If this is not the case it is generally easy to find a new matrix with full column rank that spans the same column space. The transformation $A_{t}^{L}$ is applied to $y_{t}\left(o_{t}, o_{t-1}\right)$ only and does not require to consider the elements of $\dot{\alpha}_{t}$ associated with $u_{t}$ since they do not affect $y_{t}\left(o_{t}, o_{t-1}\right)$. We can extend the transformation towards $y_{t}\left(o_{t}, m_{t-1}\right)$ but this will not lead to further reductions $y_{t}\left(o_{t}, m_{t-1}\right)$.

Define the matrix

$$
A_{t}=\left[\begin{array}{c}
A_{t}^{L} \\
A_{t}^{H}
\end{array}\right]
$$

where $A_{t}^{H}$ is chosen such that $A_{t}^{L} V_{t} A_{t}^{H \prime}=0$ and $A_{t}$ is a full rank matrix. The state space model for the transformed observation vector $A_{t} y_{t}\left(o_{t}, o_{t-1}\right)$ is given by

$$
\left(\begin{array}{c}
A_{t}^{L} y_{t}\left(o_{t}, o_{t-1}\right) \\
A_{t}^{H} y_{t}\left(o_{t}, o_{t-1}\right)
\end{array}\right)=\left(\begin{array}{c}
A_{t}^{L} c_{t}^{o} \\
A_{t}^{H} c_{t}^{o}
\end{array}\right)+\left[\begin{array}{cccc}
C_{t}^{\prime} S & 0 & 0 & 0 \\
0 & 0 & 0 & 0
\end{array}\right] \dot{\alpha}_{t}+A_{t} \varepsilon_{t}\left(o_{t}, o_{t-1}\right)
$$

where $q \times p$ matrix $S$ is defined in (2) and $\operatorname{Var}\left[A_{t} \varepsilon_{t}\left(o_{t}, o_{t-1}\right)\right]$ is a block-diagonal variance matrix with the upper-block given by $\operatorname{Var}\left[A_{t}^{L} \varepsilon_{t}\left(o_{t}, o_{t-1}\right)\right]=I$. It follows that we can remove $A_{t}^{H} y_{t}\left(o_{t}, o_{t-1}\right)$ for the application of the KFS and for the same reasons as discussed in section 4.2. In particular, for the application of KFS we can replace (9) by the two observation equations

$$
\left(\begin{array}{c}
A_{t}^{L} y_{t}\left(o_{t}, o_{t-1}\right) \\
y_{t}\left(o_{t}, m_{t-1}\right)
\end{array}\right)=\left(\begin{array}{c}
A_{t}^{L} c_{t}^{o} \\
0
\end{array}\right)+\left[\begin{array}{cccc}
C_{t}^{\prime} S & 0 & 0 & 0 \\
Z_{t}^{o m} & I & 0 & 0
\end{array}\right] \dot{\alpha}_{t}+\left(\begin{array}{c}
A_{t}^{L} \varepsilon_{t}\left(o_{t}, o_{t-1}\right) \\
0
\end{array}\right)
$$

where $Z_{t}^{o m}=\left\{Z\left(o_{t}, m_{t-1} ; \cdot\right), 0\right\}$. In most cases, the observation vector dimension of the collapsed model will be much lower than the dimension of $y_{t}^{o}$. However, when $y_{t}$ does contain many missing observations, it may become the case that the dimension of $y_{t}\left(o_{t}, o_{t-1}\right)$ is lower than $2 p$. In this case no computational gain can be achieved by transforming the model. The state space model (13) and (10) satisfies the conditions required for the KFS devices discussed in Koopman and Durbin (2003).

In case both observation vectors $y_{t}$ and $y_{t-1}$ contain no missing entries, we can apply the time-invariant transformation as developed in section 4.2 and based on the state space formulation A of section 2.2. We only require the modifications for collapsing the observation vector when missing entries in the observation vectors $y_{t}$ or $y_{t-1}$ are present. 


\subsection{Log-likelihood evaluation}

For a set of realizations $y_{1}, \ldots, y_{n}$ as generated by the state space model, we define the log-likelihood function by

$$
\ell(y)=\log p\left(y_{1}^{o}, \ldots, y_{n}^{o} ; \theta\right), \quad y=\left\{y_{t}^{o}\right\}_{t=1}^{n},
$$

where $p(\cdot)$ is the Gaussian density function, $y$ is the set of observed data, and $\theta$ is the vector of parameters introduced in section 2. The prediction error decomposition result of Schweppe (1965) implies that $\log p\left(y_{1}^{o}, \ldots, y_{n}^{o} ; \theta\right)=\log p\left(y_{1}^{o} ; \theta\right)+\sum_{t=2}^{n} \log p\left(y_{t}^{o} \mid Y_{t-1} ; \theta\right)$ where $p\left(y_{t}^{o} \mid Y_{t-1} ; \theta\right)$ can be evaluated by the Kalman filter.

Jungbacker and Koopman (2008) argue that the likelihood function $\ell(y)$ can be obtained by the Kalman filter applied to the collapsed data vector. In our case, we can limit the application of the Kalman filter to the observation equation (13). The log-likelihood function is then evaluated by

$$
\ell(y)=\text { constant }+\ell\left(y^{L}, y^{o m}\right)+\ell\left(y^{H}\right)
$$

where

$$
y^{L}=\left\{A_{t}^{L} y_{t}\left(o_{t}, o_{t-1}\right)\right\}_{t=1}^{n}, \quad y^{o m}=\left\{y_{t}\left(o_{t}, m_{t-1}\right)\right\}_{t=1}^{n}, \quad y^{H}=\left\{A_{t}^{H} y_{t}\left(o_{t}, o_{t-1}\right)\right\}_{t=1}^{n},
$$

and the constant does not depend on $\theta$ nor on the obervations. The log-likelihood function $\ell\left(y^{L}, y^{o m}\right)$ is obtained from the Kalman filter applied to the state space model (13) and (10). The log-likelihood function $\ell\left(y^{H}\right)$ can be evaluated by

$$
\ell\left(y^{H}\right)=\text { constant }-\frac{1}{2} \sum_{t=1}^{n} \log \left|V_{t}\right|-\frac{1}{2} \sum_{i=1}^{n} e_{t}^{\prime} V_{t}^{-1} e_{t}
$$

where $e_{t}=\left(I-V_{t} A_{t}^{L^{\prime}} A_{t}^{L}\right)\left[y_{t}\left(o_{t}, o_{t-1}\right)-\phi_{t}^{o} y_{t-1}\left(o_{t}, o_{t-1}\right)\right]$, for $t=1, \ldots, n$, see Jungbacker and Koopman (2008, Lemma 2).

\section{Maximum likelihood estimation}

The estimation of $\theta$ discussed in section 2 by maximum likelihood methods requires solving a potentialy high dimensional optimization problem. It involves the maximization of $\ell(y ; \theta)$ with respect to $\theta$ and where $\ell(y ; \theta)$ can be evaluated by the methods described in section 4.4 . 


\subsection{Direct maximization}

In general, large-scale optimization problems are handled by quasi-Newton algorithms as described in Nocedal and Wright (1999). These algorithms require the evaluation of $\ell(y ; \theta)$ and the score function at each iteration of the algorithm. Since the number of parameters in dynamic factor models are typically large, the numerical computation of the score function is not feasible. Fortunately, analytic expressions for the score function are available for dynamic factor models in state space form and which can be computed efficiently, see the discussion in Koopman and Shephard (1992) and Jungbacker and Koopman (2008).

Let $Q\left(\theta \mid \theta^{*}\right)$ denote the complete expected loglikelihood defined by

$$
Q\left(\theta \mid \theta^{*}\right)=\mathbb{E}_{\theta^{*}}\left[\log p\left(y^{o}, \dot{\alpha}_{1}, \ldots, \dot{\alpha}_{n} ; \theta\right) \mid y^{o}\right]
$$

where $p\left(y^{o}, \dot{\alpha}_{1}, \ldots, \dot{\alpha}_{n} ; \theta\right)$ is the joint density of $y^{o}$ and $\dot{\alpha}_{1}, \ldots, \dot{\alpha}_{n}$ for parameter vector $\theta$. The subscript $\theta^{*}$ in $\mathbb{E}_{\theta^{*}}$ emphasizes that the expectation is calculated for a given parameter vector $\theta^{*}$. From the results in Louis (1982) and Ruud (1991), we have

$$
\left.\frac{\partial Q\left(\theta \mid \theta^{*}\right)}{\partial \theta}\right|_{\theta=\theta^{*}}=\left.\frac{\partial \ell(y ; \theta)}{\partial \theta}\right|_{\theta=\theta^{*}}
$$

provided that $\theta$ is the true parameter. Note that $\ell(y ; \theta)$ is defined in (14). In Appendix B we provide the expressions for the derivatives of $Q\left(\theta \mid \theta^{*}\right)$ with respect to the system matrices of the state space model (9) and (10). From these expressions and the chain rule we can determine the score function for all the parameters in the model of section 2 . The computation of the score in this way only requires a single run of the KFS and can be done computationally efficiently due to the results of the previous section.

\subsection{Expectation-Maximization algorithm}

An alternative method is the expectation-maximization (EM) algorithm for finding the maximum likelihood estimators, see Watson and Engle (1983) and Shumway and Stoffer (1982). The EM algorithm produces a sequence of proposals $\theta^{(1)}, \theta^{(2)}, \ldots$ for the maximum likelihood estimator of $\theta$ by repeating the following two steps for $j=1,2, \ldots$ :

- E-step: obtain the expected complete likelihood $Q\left(\theta \mid \theta^{(j)}\right)$ where $\theta^{(j)}$ is the current estimate.

- M-step: maximize $Q\left(\theta \mid \theta^{(j)}\right)$ with respect to $\theta$ and set $\theta^{(j+1)}$ to the parameter value where this optimum is attained.

The EM algorithm has the attractive property that it always converges to a (local) optimum and the likelihood is ensured to increase with each iteration. The E-step can be performed by 
means of the KFS in a relatively straightforward way for the dynamic factor model considered in this paper. The maximization in the M-step can however not be done analytically. Watson and Engle (1983) propose an ad-hoc iterative scheme for the M-step. Alternatively, we can perform the M-step via a quasi-Newton scheme. Since the gradient of $Q\left(\theta \mid \theta^{(j)}\right)$ is available analytically, the necessary computations can be done computationally efficient.

\section{Computational costs and gains}

In this section we explore the computational gains that we can obtain using our new new state space specification of section 3.2 when applying the Kalman filter and associated smoothing algorithm (KFS). We do this by considering the computational costs implied by our new specification compared to those of the state space formulations A and B. The KFS method is key to both the direct maximization of the likelihood function and the E-M algorithm. In the former case it provides the likelihood function and the quantities to compute the score vector while in the latter case it provides the inputs for the Expectation step.

Below we will refer to formulations A and B from section 2.2; our new model formulation in section 3.2 will be referred to as formulation C. In comparison with A, the computing times for KFS without the presence of missing data will be the same since the two specifications are equivalent in this case. When missing observations are present, formulation $\mathrm{A}$ is not valid while formulation $\mathrm{C}$ is. The costs of the additional computations are modest when the number of missing entries is modest. When $y_{t}$ and $y_{t-1}$ have a total of $m$ unique missing entries (the entries that are both missing in $y_{t}$ and $y_{t-1}$ are counted once), the state vector $\dot{\alpha}_{t}$ needs to be increased by $m$ (temporarily). This will slow down the KFS computations but it will lead to exact results while formulation A cannot deal with missing entries. The increase in computing time depends on the number of missings in the data set.

In Table 1(a) we provide some indications of the computational costs for the dynamic factor model (1) with two dynamic factors $(q=2)$ which are modelled as stationary vector autoregressive processes. The comparisons are carried out for three different observation vector dimensions $N=10,50,100$. We have implemented the collapsed KFS as described in section 4.3. The computations are carried out for $n=1000$ observations and the reported relative times are based on the recorded computing times for the full sample of $y_{1}, \ldots, y_{n}$ in which randomly chosen entries are treated as missing. Our results reveal, for example, that for $N=50$ and for $1 \%$ missing observations, the computations take 1.5 times longer than those for formulation A (instead of, say, 20 seconds, it takes 30 seconds). When the number of missings has increased by $10 \%$, the computations take 2.6 times longer.

The formulation $\mathrm{B}$ also provides exact results when data is missing and it is the approach adopted by Banbura and Modugno (2010). However, we have argued in this paper that formulation $\mathrm{C}$ is computationally more efficient. In Table $1(\mathrm{~b})$ we compare the computing 
times for formulations B and C. The gains of the new formulation compared to B are quite considerable. For the same model as described above with $N=50$, the KFS for formulation $\mathrm{C}$ is almost 88 times faster when we have 1\% missings while it is 44 times faster when $10 \%$ of the data is missing. These gains are considerable and they are even higher and more dramatic when $N$ increases to higher values. We therefore suggest to use formulation $\mathrm{C}$ for dynamic factor analysis in the presence of missing data.

\section{[ Table 1 about here. ]}

\section{Conclusions}

Dynamic factor analysis has been given renewed attention in the economics and finance literature recently. High-dimensional dynamic models with multiple factors contain many parameters that need to be estimated. In case maximum likelihood estimation is requested and optimization is employed via Fisher-scoring and/or the EM algorithm, computational efficient methods are of key importance. Various problems arise when these methods are used when missing entries in the data set are present. A standard solution places the full idiosyncratic component vector into the state vector. It will lead to a high-dimensional state vector for a model with a high-dimensional observation vector. The associating computations for Kalman filtering and smoothing will slow down considerably. To circumvent this problem, we propose a new state space formulation of the model that allows for missing values and can exploit existing devices for computational efficiency. Only the idiosyncratic components associated with missing entries for the concurrent and previous time periods are accommodated in the state vector, all other ones are removed from the state vector. As a result, the dimension of the state vector is kept to a minimum. Also we have shown that the existing computational devices to accelerate the computations can be adopted. In our formulation the dimension of the state vector varies over time so the implementation of the Kalman filter and smoothing methods requires attention. However, we can obtain high computational savings even when the number of missing entries is moderate, see Table 1.

\section{Acknowledgment}

We would like to thank the Editor Christopher Otrok and referees for their comments on an earlier version of the paper. Michel van der Wel acknowledges the support from CREATES at the University of Aarhus and funded by the Danish National Research Foundation 


\section{Appendix A : A dynamic factor state space model}

Consider the dynamic factor model with the $q$ factors in $f_{t}$ following a vector autoregressive moving average process. An example of a dynamic process for $f_{t}$ is given by

$$
f_{t+1}=\Phi_{1} f_{t}+\Phi_{2} f_{t-1}+\eta_{t}+\Theta_{1} \eta_{t-1}, \quad \eta_{t} \sim N\left(0, \Sigma_{\eta}\right), \quad t=1, \ldots, n,
$$

where the $q \times q$ autoregressive coefficient matrices $\Phi_{1}$ and $\Phi_{2}$ and the $q \times q$ moving average coefficient matrix $\Theta_{1}$ are restricted such that $f_{t}$ is a stationary process. This process can be represented in the general formulation (2) and (3), with the state vector $\alpha_{t}$ of dimension $p=2 \cdot q$ and the disturbance vector $\eta_{t}$ of dimension $k=q$, by having the state vector and the system matrices equal to

$$
\alpha_{t}=\left(\begin{array}{c}
f_{t} \\
\Phi_{2} f_{t-1}+\Theta_{1} \eta_{t-1}
\end{array}\right), \quad S=\left[\begin{array}{ll}
I_{q} & 0_{q \times q}
\end{array}\right], \quad T=\left[\begin{array}{cc}
\Phi_{1} & I_{q} \\
\Phi_{2} & 0_{q \times q}
\end{array}\right], \quad R=\left[\begin{array}{c}
I_{q} \\
\Theta_{1}
\end{array}\right] .
$$

The observations rely on the factors, or implicitly on the state vector, via the relations

$$
y_{t}=\Lambda f_{t}+\varepsilon_{t}=Z \alpha_{t}+\varepsilon_{t}, \quad t=1, \ldots, n
$$

where $Z=\Lambda S$.

\section{Appendix B : the details for score evaluation}

Suppose the vector time series $y_{1}, \ldots, y_{n}$ is generated by the linear Gaussian state space model as given by

$$
\begin{aligned}
y_{t} & =c_{t}+Z_{t} \alpha_{t}+\varepsilon_{t} & \varepsilon_{t} & \sim N\left(0, H_{t}\right), \\
\alpha_{t+1} & =d_{t}+T_{t} \alpha_{t}+R_{t} \eta_{t} & & \eta_{t} \sim N\left(0, Q_{t}\right),
\end{aligned}
$$

with $\alpha_{1} \sim N\left(a_{1 \mid 0}, P_{1 \mid 0}\right)$ and $t=1, \ldots, n$. The system vectors and matrices $c_{t}, d_{t}, Z_{t}, T_{t}, H_{t}$ and $Q_{t}$ are fixed and may depend on the parameter vector $\theta$. Selection matrix $R_{t}$ has full column rank, is fixed and does not depend on $\theta$. Finally, we assume that $H_{t}$ is a non-singular variance matrix. Let

$$
a_{t \mid n}=\mathbb{E}\left(\alpha_{t} \mid y_{1}, \ldots, y_{n}\right), \quad P_{t \mid n}=\operatorname{Var}\left(\alpha_{t} \mid y_{1}, \ldots, y_{n}\right), \quad P_{t+1, t \mid n}=\operatorname{Cov}\left(\alpha_{t+1}, \alpha_{t} \mid y_{1}, \ldots, y_{n}\right)
$$

for $t=1, \ldots, n$. These quantities can be computed by the Kalman filter and associating state smoothing method, see Durbin and Koopman (2001, Chapter 4). Denote $\ell(y)$ by the $\log$-likelihood of $y_{1}, \ldots, y_{n}$. We then have the following expressions for the score vectors with 
respect to each of the system matrices

$$
\begin{aligned}
\frac{\partial \ell(y)}{\partial d_{t}}=\bar{R}_{t}^{\prime} Q_{t}^{-1} \bar{R}_{t}\left(a_{t+1 \mid n}-T_{t} a_{t \mid n}-d_{t}\right), & \frac{\partial \ell(y)}{\partial T_{t}}=\bar{R}_{t}^{\prime} Q_{t}^{-1} \bar{R}_{t}\left(M_{T_{t}}-T_{t} M_{Z_{t}}\right) \\
\frac{\partial \ell(y)}{\partial c_{t}}=H_{t}^{-1}\left(y_{t}-Z_{t} a_{t \mid n}-c_{t}\right), & \left.\frac{\partial \ell(y)}{\partial Z_{t}}=H_{t}^{-1}\left[\left(y_{t}-c_{t}\right) a_{t \mid n}^{\prime}-Z_{t} M_{Z_{t}}\right)\right], \\
\frac{\partial \ell(y)}{\partial Q_{t}}=Q_{t}^{-1} M_{Q_{t}} Q_{t}^{-1}-\frac{1}{2} \operatorname{diag}\left\{Q_{t}^{-1} M_{Q_{t}} Q_{t}^{-1}\right\}, & \\
\frac{\partial \ell(y)}{\partial H_{t}}=H_{t}^{-1} M_{H_{t}} H_{t}^{-1}-\frac{1}{2} \operatorname{diag}\left\{H_{t}^{-1} M_{H_{t}} H_{t}^{-1}\right\}, &
\end{aligned}
$$

where $\bar{R}_{t}=\left(R_{t}^{\prime} R_{t}\right)^{-1} R_{t}^{\prime}$ and

$$
\begin{array}{ll}
M_{Q_{t}}=\mathbb{E}\left(\eta_{t} \eta_{t}^{\prime} \mid y_{1}, \ldots, y_{n}\right)-Q_{t}, & M_{T_{t}}=a_{t+1 \mid n} a_{t \mid n}^{\prime}+P_{t+1, t \mid n} \\
M_{H_{t}}=\left(y_{t}-Z_{t} a_{t \mid n}\right)\left(y_{t}-Z_{t} a_{t \mid n}\right)^{\prime}+Z_{t} P_{t \mid n} Z_{t}^{\prime}-H_{t}, & M_{Z_{t}}=a_{t \mid n} a_{t \mid n}^{\prime}+P_{t \mid n}
\end{array}
$$

The matrix $M_{Q_{t}}$ can be evaluated using the identity $\eta_{t}=\bar{R}_{t}\left(\alpha_{t+1}-T_{t} \alpha_{t}-d_{t}\right)$ and from $P_{t+1, t \mid n}$ and $P_{t \mid n}$. 


\section{References}

Anderson, B. D. O. and J. B. Moore (1979). Optimal Filtering. Englewood Cliffs: PrenticeHall.

Bai, J. (2003). Inferential theory for factor models of large dimensions. Econometrica 71, $135-72$.

Banbura, M. and M. Modugno (2010). Maximum likelihood estimation of large factor model on datasets with arbitrary pattern of missing data. ECB Working Paper Series 1189.

Dempster, A. P., N. Laird, and D. B. Rubin (1977). Maximum likelihood from incomplete data via the EM algorithm. J. Royal Statistical Society B 39, 1-38.

Doz, C., D. Giannone, and L. Reichlin (2010). A quasi maximum likelihood approach for large approximate dynamic factor models. Rev. Economics and Statistics, forthcoming.

Duncan, D. B. and S. D. Horn (1972). Linear dynamic recursive estimation from the viewpoint of regression analysis. J. American Statistical Association 67, 815-21.

Durbin, J. and S. J. Koopman (2001). Time Series Analysis by State Space Methods. Oxford: Oxford University Press.

Engle, R. F. and M. W. Watson (1981). A one-factor multivariate time series model of metropolitan wage rates. J. American Statistical Association 76, 774-81.

Forni, M., M. Hallin, M. Lippi, and L. Reichlin (2000). The generalized dynamic factor model: Identification and estimation. Rev. Economics and Statistics 82, 540-54.

Geweke, J. (1977). The dynamic factor analysis of economic time series. In D. J. Aigner and A. S. Goldberger (Eds.), Latent variables in socio-economic models. Amsterdam: North-Holland.

Harvey, A. C. (1989). Forecasting, Structural Time Series Models and the Kalman Filter. Cambridge: Cambridge University Press.

Jungbacker, B. and S. J. Koopman (2008). Likelihood-based analysis for dynamic factor models. Working paper.

Kapetanios, G. and M. Marcellino (2009). A parametric estimation method for dynamic factor models of large dimensions. J. Time Series Analysis 30, 208-38.

Koopman, S. J. and J. Durbin (2003). Filtering and smoothing of state vector for diffuse state space models. J. Time Series Analysis 24, 85-98.

Koopman, S. J. and N. Shephard (1992). Exact score for time series models in state space form. Biometrika 79, 823-6. 
Louis, T. A. (1982). Finding observed information using the EM algorithm. J. Royal Statistical Society B 44, 98-103.

Nocedal, J. and S. J. Wright (1999). Numerical Optimization. New York: Springer Verlag.

Otrok, C. and P. M. Pourpourides (2008). On the cyclicality of real wages and wage differentials. Working paper.

Otrok, C. and C. H. Whiteman (1998). Bayesian leading indicators: Measuring and predicting economic conditions in Iowa. International Economic Review 39, 997-1014.

Reis, R. and M. W. Watson (2010). Relative goods' prices, pure inflation, and the phillips correlation. American Economic Journal Macroeconomics 2, 128-57.

Ruud, P. (1991). Extensions of estimation methods using the EM algorithm. J. Econometrics 49, 305-41.

Sargent, T. J. and C. A. Sims (1977). Business cycle modeling without pretending to have too much a priori economic theory. In C. A. Sims et al. (Eds.), New methods in business cycle research. Minneapolis: Federal Reserve Bank of Minneapolis.

Schweppe, F. (1965). Evaluation of likelihood functions for Gaussian signals. IEEE Transactions on Information Theory 11, 61-70.

Shumway, R. H. and D. S. Stoffer (1982). An approach to time series smoothing and forecasting using the EM algorithm. J. Time Series Analysis 3, 253-64.

Shumway, R. H. and D. S. Stoffer (2000). Time Series Analysis and Its Applications. New York: Springer-Verlag.

Stock, J. H. and M. Watson (2002a). Forecasting using principal components from a large number of predictors. J. American Statistical Association 97, 1167-79.

Stock, J. H. and M. Watson (2002b). Macroeconomic forecasting using diffusion indexes. J. Business and Economic Statist. 20, 147-162.

Watson, M. W. and R. F. Engle (1983). Alternative algorithms for the estimation of dynamic factor, MIMIC and varying coefficient regression. J. Econometrics 23, 385400. 


\section{TABle 1: Computational COSTS And Gains}

The panel (a) presents ratios of computing times for the formulation of section 3.2 with missing data divided by those for the formulation without missing data (this is formulation A of section 2.2). For example, the value 2 indicates that the computational demands are twice as high. The panel (b) presents ratios of computing times for the formulation $\mathrm{B}$ of section 2.2 divided by those for the formulation of section 3.2 with missing data. For example, the value 2 indicates that the new device is twice as fast. The ratios are presented for different dimensions $N$ of observation vector $y_{t}$ and for different percentages of missing data.

\begin{tabular}{lrrr}
\multicolumn{3}{c}{ (a) } & Costs relative to A \\
\hline$N$ & \multicolumn{3}{c}{ percentage missing } \\
& $1 \%$ & $10 \%$ & $25 \%$ \\
\hline 10 & 1.4 & 1.8 & 2.3 \\
50 & 1.5 & 2.6 & 8.9 \\
100 & 1.2 & 3.9 & 24.8 \\
\hline
\end{tabular}

\begin{tabular}{lrrr}
\multicolumn{4}{c}{ (b) Gains relative to B } \\
\hline$N$ & \multicolumn{3}{c}{ percentage missing } \\
& $1 \%$ & $10 \%$ & $25 \%$ \\
\hline 10 & 2.1 & 1.5 & 1.1 \\
50 & 87.9 & 43.7 & 11.3 \\
100 & 625.5 & 197.5 & 25.8 \\
\hline
\end{tabular}

